\title{
Evaluation de la diversité agromorphologique des descendances hybrides de cocotier (Cocos nucifera L.) Nain Jaune Malaisie x Grand Vanuatu en Côte d'Ivoire
}

\author{
Zadjéhi Eric-Blanchard KOFFI ${ }^{1,2}$, Jean Louis Konan KONAN ${ }^{1 *}$, \\ Auguste Emmanuel ISSALI ${ }^{1}$, Thierry Tacra LEKADOU ${ }^{1}$, Roland BOURDEIX ${ }^{3}$, \\ René Kouassi ALLOU ${ }^{1}$ et Irié Arsène ZORO BI ${ }^{2}$ \\ ${ }^{1}$ Centre National de Recherche Agronomique (CNRA), Station de Recherche Marc Delorme, 07 BP 13 Abidjan \\ 07, Côte d'Ivoire. \\ ${ }^{2}$ Laboratoire de Génomique Fonctionnelle et d'Amélioration Génétique, UFR des Sciences de la Nature, \\ Université d'Abobo-Adjamé, 02 BP 801 Abidjan 02, Côte d'Ivoire. \\ ${ }^{3}$ Centre de coopération Internationale en recherche agronomique pour le développement (CIRAD). Boulevard \\ de la Lironde, TA80/PS3, 34398 Montpellier, Cedex 5 France. \\ *Auteur correspondant, E-mail: konankonanjeanlouis@yahoo.fr; zadjehi2003@yahoo.fr; Tel: + 22505174183
}

\section{RESUME}

Ce travail vise à évaluer la diversité agromorphologique des descendances hybrides de cocotier Nain Jaune Malaisie x Grand Vanuatu tolérants à la maladie du jaunissement. Les observations ont porté sur 30 descripteurs liés aux stipes, feuilles, inflorescences et fruits. Les résultats montrent que 12 descripteurs permettent de discriminer les individus au niveau inter et intra-descendance. La structuration de ces descendances a révélé deux groupes. Il s'agit des descendances d15 qui se sont distinguées des autres. Les arbres de la descendance d15 sont caractérisés par une Circonférence du stipe à $20 \mathrm{~cm}$ du sol $(134,52 \mathrm{~cm})$, un Epaisseur de Pétiole (3,25 cm), une Largeur de Foliole $(6,42 \mathrm{~cm})$, un Nombre d'Epillets (40), un Nombre d'Epillet Sans Fleur femelle (23), un Diamètre polaire de la noix $(20,03 \mathrm{~cm})$ et une masse de la bourre $(291,82$ g) supérieures à ceux des autres descendances. Le Géniteur G15 qui donne des descendances robustes et présentant des aptitudes favorables à une bonne production est à sélectionner pour produire des hybrides NJM x GVT tolérants et haut producteurs.

(C) 2013 International Formulae Group. All rights reserved.

Mots clés: Cocotier, hybride, jaunissement mortel, diversité agromorphologique.

\section{INTRODUCTION}

Le cocotier (Cocos nucifera L.) est un oléagineux pérenne présent dans toute la zone intertropicale. Toutes les parties de l'arbre sont valorisées de manière artisanale et/ou industrielle (Bourdeix et al., 2005). La cocoteraie mondiale couvre une superficie de
11,8 millions d'hectares (Van der Vossen et Chipungahelo, 2007) et environ 10 millions de familles vivent de sa culture. En Côte d'Ivoire, celle-ci couvre une superficie de 50000 hectares et est la principale source de revenus de plus de 20 mille familles de la basse Côte d'Ivoire (Assa et al., 2006). 
Aujourd'hui, sous la pression des différents ravageurs et maladies, la superficie mondiale de la cocoteraie est en pleine réduction. Parmi les maladies, le jaunissement mortel représente l'une des plus dévastatrices des cocoteraies (Van der Vossen et Chipungahelo, 2007). En effet, la maladie a déjà détruit des milliers d'hectares de cocoteraie dans plusieurs régions $\mathrm{du}$ monde telles que l'Afrique, les Caraïbes et l'Amérique Centrale (Baudouin et al., 2009). Elle représente le risque phytosanitaire le plus important auquel est confronté la nuciculture. La maladie se manifeste d'abord par une chute prématurée des fruits, ensuite le jaunissement et le dessèchement des extrémités des épillets des inflorescences qu'elles soient ouvertes ou enfermées dans la spathe, suivie du jaunissement et de la mort des feuilles (Oropeza et al., 2005). L'arbre meurt 3 à 7 mois après l'apparition des premiers symptômes. La recherche n'a pas encore mis au point un traitement chimique efficace contre le phytoplasme responsable de la maladie. La lutte génétique, à travers la création de variétés résistantes ou tolérantes demeure donc la solution la plus efficiente. Des tests de résistance on été ainsi conduits en zone endémique ghanéenne depuis 1981 par le Centre National de Recherche Agronomique (CNRA), en collaboration avec l'Oil Palm Research Institute ou OPRI (Sangaré et al., 1992 ; Konan, 2005). A l'issue de ces tests, le cultivar Grand de Vanuatu (GVT) provenant de la collection de Côte d'Ivoire a été identifié comme source de tolérance à la maladie. Pour diversifier les sources de tolérance et pour une amélioration du niveau de tolérance, des descendances hybrides issues de croisements entre le Nain Jaune Malaisie (NJM) et le Grand de Vanuatu (GVT) ont été crées et plantés simultanément en Côte d'Ivoire et au Ghana en 1998. L'évaluation épidémiologique par rapport à la maladie au Ghana par Dery et al. (2005) et Quaicoe et al. (2009) a montré une variabilité dans le comportement de ces descendances. Par contre, l'évaluation agromorphologique de ces hybrides n'est pas encore faite. La présente étude vise l'évaluation de la diversité agromorphologique de 18 descendances hybrides plantées en Côte d'Ivoire afin de donner des indications aux sélectionneurs quant aux choix des géniteurs appropriés pour la création d'hybride tolérant au jaunissement mortel et susceptible d'une bonne production.

\section{MATERIEL ET METHODES}

\section{Site d'étude et matériel végétal}

L'essai est abrité par la parcelle 034 de la station de recherche Marc Delorme de PortBouët $\left(5^{\circ} 14^{\prime}\right.$ et $5^{\circ} 15^{\prime}$ de latitude Nord et $3^{\circ} 54^{\prime}$ et $3^{\circ} 55^{\prime}$ de longitude Ouest) Abidjan, dans le sud de la Côte d'Ivoire (Figure 1). Le climat de la région sud de la Côte d'Ivoire comprend quatre saisons dont deux pluvieuses (avril à juillet et octobre à novembre) et deux sèches (décembre à mars et août à septembre). La température moyenne varie entre $24,50{ }^{\circ} \mathrm{C}$ et $27,73{ }^{\circ} \mathrm{C}$. L'insolation totale par année atteint 2238,3 heures avec une humidité moyenne de $86,02 \%$. La pluviométrie est caractérisée par une précipitation moyenne annuelle de 1673,99 mm. Le sol de la station Marc Delorme est constitué de sables tertiaires (Tié bi, 1984).

Le matériel végétal est constitué de 18 descendances hybrides (codé de d1 à d18) issues de croisements entre Nain Jaune Malaisie (NJM) et Grand Vanuatu (GVT). Ces croisements ont impliqué 18 génotypes géniteurs mâles GVT (G1 à G18) et un génotype parent femelle NJM. Les hybrides ont été plantés en 1998.

\section{Dispositif expérimental}

Les arbres on été plantés selon un dispositif en blocs complets randomisés avec 6 répétitions. Chaque bloc a été composé de 10 lignes de 12 arbres chacune. Les lignes 1 et 32 ainsi que les arbres 1 et 26 ont constitué les bordures composées d'une descendance NJM x GVT. La densité de plantation a été de 160 arbres à l'hectare. Au total 832 arbres ont été plantés. La superficie de la parcelle est donc de 5,2 hectares. 


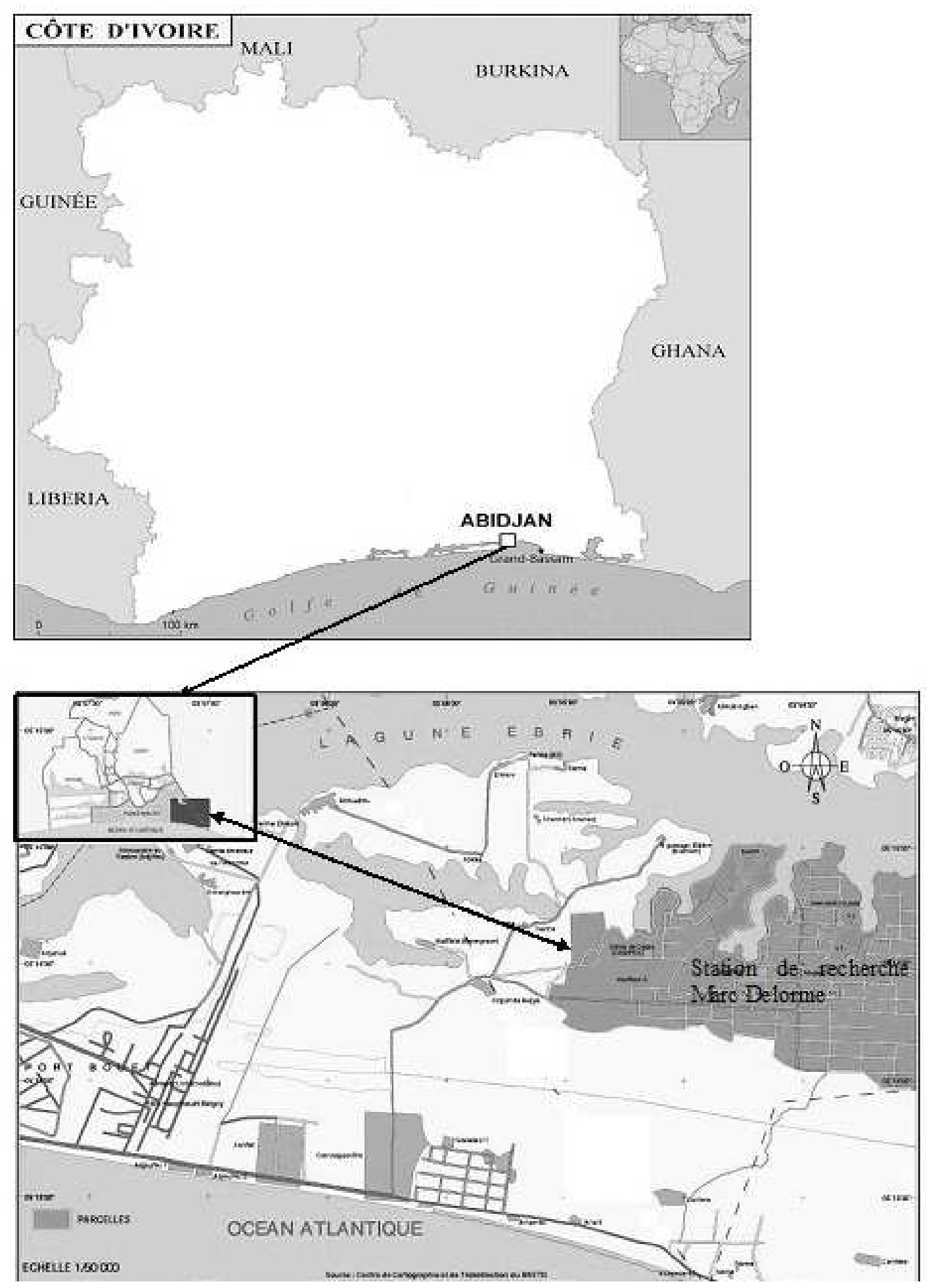

Figure 1 : Situation géographique du site d'étude. 


\section{Descripteurs agromorphologiques}

Trois arbres ont été choisis de manière aléatoire par bloc et par descendance. Soit 18 arbres par descendance et un total de 324 arbres étudiés pour les 18 croisements mis en comparaison. Les mesures ont porté sur le stipe, les feuilles, les inflorescences et les fruits. Trente descripteurs ont permis d'effectuer une structuration des différentes descendances testées (Tableau 1).

\section{Analyses statistiques}

Les logiciels SPSS 16.0 et STATISTICA 7.1 ont été utilisés. Une analyse de variance incorporant la comparaison des moyennes selon Newman et Keuls au seuil de $5 \%$ a été réalisée. Les coefficients de variation ont permis de vérifier la variabilité intradescendance au seuil de $20 \%(0,20)$. Une Analyse en Composante Principale a été réalisée pour rechercher les facteurs impliqués

Tableau 1 : Récapitulatif des variables mesurées sur les descendances hybrides de cocotier NJM x GVT.

\begin{tabular}{|c|c|}
\hline Désignation & Variables observées \\
\hline \multirow{4}{*}{ Stipe } & -Hauteur (HS) \\
\hline & -Circonférence à 20 cm du sol (C20) \\
\hline & -Circonférence à 150 cm du sol (C150) \\
\hline & -Longueur sur 11 cicatrices foliaires (L11CF) \\
\hline \multirow{8}{*}{ Feuilles } & -Longueur du rachis (LR) \\
\hline & -Longueur du pétiole (LP) \\
\hline & -Epaisseur du pétiole (EP) \\
\hline & -Largeur du pétiole (LGP) \\
\hline & -Nombre de folioles sur un coté de la palme (NBF) \\
\hline & -Longueur de la foliole (LF) \\
\hline & -Largeur de la foliole (LGF) \\
\hline & -Surface foliaire théorique (SF=2 x NBF x LF x LGF) \\
\hline \multirow{8}{*}{ Inflorescences } & -Longueur de l'axe (ILA) \\
\hline & -Longueur du pédoncule (ILP) \\
\hline & -Longueur de l’épillet (ILE) \\
\hline & -Longueur entre le point d'attache de l'épillet et celui du premier fruit (ILPRF) \\
\hline & -Nombre d'épillets (NBE) \\
\hline & -Nombre d'épillets portant des fleurs femelles (NBEF) \\
\hline & -Nombre d'épillets sans fleurs femelles (NBESF) \\
\hline & -Nombre de fleurs femelles (NBFF) \\
\hline \multirow{10}{*}{ Fruits } & -Diamètre Polaire (DP) \\
\hline & -Diamètre Equatorial (DE) \\
\hline & -Diamètre polaire sur diamètre équatorial (DP /DE) \\
\hline & -Masse de la noix (MN) \\
\hline & -Masse de la bourre (MB) \\
\hline & -Masse de la coque (MC) \\
\hline & -Masse de l'amande (MA) \\
\hline & -Masse de l'eau (ME) \\
\hline & -Masse du coprah par noix (MCP) \\
\hline & -Pourcentage de coprah par noix (Q) \\
\hline
\end{tabular}


dans la variabilité des descendances. Les données ont été préalablement centrées et réduites. Pour structurer les individus analysés, une Classification Ascendante Hiérarchique (CAH) a été réalisée. Cette classification a été faite sur la base des distances euclidiennes utilisant comme option d'agrégation, la méthode des moyennes non pondérées des groupes associés ou UPGMA (Unweighted Pair-Group Method using Arithmetic average) (Baudouin et Santos, 2005). Les tests du Lambda de Wilk et de Fisher ont été utilisés pour évaluer les distances de Mahalanobis entre les classes mises en évidence par la $\mathrm{CAH}$, en utilisant la méthode de l'analyse discriminante. Ces deux tests ont permis d'apprécier la structuration fournie par la classification ascendante hiérarchique.

\section{RESULTATS}

\section{Evaluation de la performance des} descripteurs

Sur 30 variables utilisées pour l'analyse de variance, sept se sont montrées discriminantes. Ainsi les variables C20 $(\mathrm{F}=2,93 ; \mathrm{P}<0,001), \mathrm{EP}(\mathrm{F}=2,34 ; \mathrm{P}=0,004)$, LGF $(\mathrm{F}=1,95 ; \mathrm{P}=0,02)$, NBE $(\mathrm{F}=1,80 ; \mathrm{P}=$ 0,04), NBESF $(\mathrm{F}=3,66 ; \quad \mathrm{P}<0,001), \quad \mathrm{DP}$ $(\mathrm{F}=2,29 ; \mathrm{P}=0,005)$ et $\mathrm{MB} \quad(\mathrm{F}=2,14 ; \mathrm{P}=$ $0,009)$ ont permis de discriminer les 18 descendances hybrides NJM x GVT. Les descendances d15 ont en moyenne les plus gros bulbes $(\mathrm{C} 20=134,52 \mathrm{~cm})$. Les autres descendances ne diffèrent pas significativement entre elles. Elles ont des moyennes de C20 qui varient de 103,82 à $119,68 \mathrm{~cm}$. La variabilité intra-descendance n'est pas élevée pour ce caractère. Les coefficients de variations sont inférieurs à 0,20 (Tableau 2).

L'épaisseur du pétiole classe les descendances en trois groupes. Un premier groupe constitué des descendances d10, d9, $\mathrm{d} 4, \mathrm{~d} 1, \mathrm{~d} 7, \mathrm{~d} 12$ qui ont des pétioles moins épais avec des valeurs comprises entre 2,97 et $3,01 \mathrm{~cm}$. Un deuxième groupe constitué par les d15 qui fournissent les pétioles les plus épais $(3,25 \mathrm{~cm})$. Un troisième groupe intermédiaire et formé par les descendances d11, d3, d14, d18, d17, d8, d5, d6, d16, d13 et d2 qui ont des épaisseurs de pétioles variant entre 3,03 et $3,12 \mathrm{~cm}$. Les folioles les moins larges sont observées chez les descendances d12 $(5,73 \mathrm{~cm})$. Les d15 ont en moyenne les plus fortes valeurs $(6,42 \mathrm{~cm})$. Les autres descendances fournissent des valeurs intermédiaires qui sont comprises entre 5,91 et $6,29 \mathrm{~cm}$. Les arbres étudiés sont relativement homogènes pour les caractères liés à la feuille. Les coefficients de variations sont inférieurs à 0,20 (Tableau 3).

Les descendances d15 ont en moyenne plus d'épillets (40) par inflorescence. Les d18 ont les plus faibles valeurs (34). Les autres descendances fournissent des nombres compris entre 36 et 39 . Les descendances d15 qui ont un nombre d'épillets sans fleurs femelles d'environ 23 sont supérieures aux d5 (12) pour ce caractère. Les autres descendances ont des valeurs intermédiaires qui varient de 13 à 20 (Tableau 4). Les coefficients de variation, relativement élevés pour le descripteur NBESF $(0,20$ à 0,45$)$ attestent d'une variabilité intra-descendance plus élevée. Le diamètre polaire de la noix est plus élevé chez d15 $(20,03 \mathrm{~cm})$ que d18 $(17,39 \mathrm{~cm})$ et $\mathrm{d} 5(17,73 \mathrm{~cm})$ qui ont les plus faibles valeurs. Ce caractère est stable, les CV sont compris entre 0,06 et 0,17 .

Les masses de bourre sont plus élevées chez d15 (291,82 g) que chez d5, d10, d18, d7, d3, d4 et d12 qui présente des valeurs plus faibles comprises entre 192,5 et $270,76 \mathrm{~g}$. Au niveau de la masse de la bourre, la descendance la plus hétérogène est le $\mathrm{d} 9$ $(\mathrm{CV}=0,43)$, quand $\mathrm{d} 5$ parait plus homogène $(\mathrm{CV}=0,15)$.

Les 23 autres variables ne permettent pas de discriminer les descendances étudiées. Par contre, parmi ces variables, HS, NBEF, NBFF, L11CF et ME ont des coefficients de variation très élevés. Ces coefficients sont tous supérieurs à 0,20 et atteignent 0,75 (Tableaux 2, 3, 5 et 6). Les 18 variables restantes ne présentent presque pas de variabilité intra et inter-descendance.

Les résultats de l'analyse en 
composantes principales (ACP) ont montré que les deux premiers axes permettent d'expliquer l'essentiel de la variabilité révélée par les variables quantitatives. Ces deux axes principaux expriment $74,99 \%$ de la variabilité totale observée (Tableau 7). L'axe 1 exprime $58,37 \%$ de la variabilité totale. Il est expliqué par les variables C20, EP, LGF, NBE, NBESF, DP, MB. L'axe 2 traduit $16,62 \%$ de la variabilité totale. Les variables EP et DP ont plus contribué à sa formation. La projection des individus dans le plan 1-2 de l'ACP qui représente $74,99 \%$ de la variabilité totale, divise les 18 descendances en 2 groupes. Le premier groupe est constitué de d15 et le deuxième des autres descendances (Figure 2). Les descendances d15 se distinguent par leur robustesse. Elles ont les valeurs les plus élevées de C20, EP, LGF, NBE, NBESF, DP, MB.

\section{Structuration de la diversité agromorphologique La Classification Ascendante}

Hiérarchique réalisée fait apparaître trois classes (Figure 3). Une première classe constituée des descendances d1, d3, d4, d5,d7, $\mathrm{d} 10, \mathrm{~d} 11, \mathrm{~d} 12, \mathrm{~d} 14, \mathrm{~d} 17$ et $\mathrm{d} 18$, une deuxième composée de d2, d6, d8, d9, d13 et d16. La troisième est constituée uniquement de $\mathrm{d} 15$. Le test du Lambda de Wilks entre les différentes classes est significatif ( $p=0,01)$. Aussi le test de Fischer indique une différence hautement significative entre la classe 3 et les autres. Cependant les classes 1 et 2 sont identiques au seuil de 5\% (Tableau 8). L'analyse discriminante vient confirmer ainsi la structuration obtenue avec l'Analyse en Composante Principale. Les arbres de la classe 3 constitués uniquement des descendances d15 se différencient par des valeurs de C20, EP, LGF, NBE, NBESF, DP, MB supérieures à celles des autres classes. Les 2 autres classes qui ne se distinguent pas les unes des autres peuvent être regroupées dans une classe unique. Cette classe est constituée d'arbres moins robustes.

Tableau 2 : Valeurs moyennes, coefficients de variations des variables agromorphologiques liées au stipe et tests de comparaison chez les hybrides de cocotier étudiés.

\begin{tabular}{llcccc}
\hline Descendances & & C20 $(\mathbf{c m})$ & C150 $(\mathbf{c m})$ & HS $(\mathbf{c m})$ & L11CF $(\mathbf{c m})$ \\
\hline d1 & Moy & $119,68 \mathrm{~b}$ & 72,85 & 359,34 & 44,47 \\
d2 & CV & 0,12 & 0,06 & 0,34 & 0,38 \\
& Moy & $114,20 \mathrm{~b}$ & 73,64 & 340,47 & 41,05 \\
d3 & CV & 0,15 & 0,05 & 0,34 & 0,34 \\
& Moy & $110,05 \mathrm{~b}$ & 71,70 & 347,21 & 42,37 \\
d4 & CV & 0,18 & 0,08 & 0,33 & 0,28 \\
& Moy & $107,50 \mathrm{~b}$ & 70,94 & 347,26 & 43,19 \\
d5 & CV & 0,16 & 0,06 & 0,40 & 0,44 \\
& Moy & $117,88 \mathrm{~b}$ & 73,35 & 350,45 & 45,49 \\
d6 & CV & 0,14 & 0,06 & 0,40 & 0,30 \\
& Moy & $112,91 \mathrm{~b}$ & 71,50 & 362,42 & 42,79 \\
d7 & CV & 0,12 & 0,07 & 0,37 & 0,34 \\
& Moy & $115,72 \mathrm{~b}$ & 71,34 & 322,62 & 43,73 \\
d8 & CV & 0,14 & 0,07 & 0,44 & 0,32 \\
& Moy & $115,42 \mathrm{~b}$ & 71,74 & 365,23 & 42,84 \\
d9 & CV & 0,14 & 0,08 & 0,05 & 0,40 \\
& Moy & $106,74 \mathrm{~b}$ & 69,77 & 321,61 & 38,08 \\
d10 & CV & 0,13 & 0,08 & 0,36 & 0,33 \\
& Moy & $112,86 \mathrm{~b}$ & 73,43 & 356,28 & 43,49
\end{tabular}




\begin{tabular}{llcccc} 
& CV & 0,16 & 0,06 & 0,43 & 0,34 \\
$\mathrm{~d} 11$ & Moy & $111,05 \mathrm{~b}$ & 73,59 & 322,73 & 39,64 \\
$\mathrm{~d} 12$ & CV & 0,17 & 0,08 & 0,47 & 0,48 \\
& Moy & $109,56 \mathrm{~b}$ & 73,95 & 326,99 & 41,60 \\
$\mathrm{~d} 13$ & CV & 0,06 & 0,05 & 0,43 & 0,29 \\
& Moy & $114,41 \mathrm{~b}$ & 74,55 & 343,86 & 43,93 \\
$\mathrm{~d} 14$ & CV & 0,14 & 0,08 & 0,44 & 0,37 \\
& Moy & $106,78 \mathrm{~b}$ & 72,33 & 328,81 & 41,45 \\
$\mathrm{~d} 15$ & CV & 0,15 & 0,09 & 0,48 & 0,39 \\
& Moy & $134,52 \mathrm{a}$ & 75,10 & 383,58 & 49,52 \\
$\mathrm{~d} 16$ & CV & 0,13 & 0,06 & 0,35 & 0,27 \\
& Moy & $103,82 \mathrm{~b}$ & 71,07 & 322,42 & 42,19 \\
$\mathrm{~d} 17$ & CV & 0,09 & 0,07 & 0,41 & 0,32 \\
& Moy & $112,17 \mathrm{~b}$ & 71,68 & 345,74 & 47,54 \\
$\mathrm{~d} 18$ & CV & 0,09 & 0,06 & 0,51 & 0,48 \\
& Moy & $109,34 \mathrm{~b}$ & 71,04 & 316,95 & 42,92 \\
& CV & 0,15 & 0,07 & 0,56 & 0,33 \\
\hline
\end{tabular}

Les moyennes indexées par la même lettre ne sont pas significativement différentes au seuil de 5\% (test de Newman Keuls).

Tableau 3: Valeurs moyennes, coefficients de variation des variables agromorphologiques liées aux feuilles et tests de comparaison chez les hybrides de cocotier étudiés.

\begin{tabular}{|c|c|c|c|c|c|c|c|c|c|}
\hline Descendances & & $\begin{array}{c}\text { LP } \\
(\mathbf{c m})\end{array}$ & $\begin{array}{l}\text { LGP } \\
\text { (cm) }\end{array}$ & $\begin{array}{c}\text { EP } \\
(\mathbf{c m})\end{array}$ & $\begin{array}{c}\text { LR } \\
(\mathbf{c m})\end{array}$ & NBF & $\begin{array}{c}\mathbf{L F} \\
(\mathbf{c m})\end{array}$ & $\begin{array}{l}\text { LGF } \\
\text { (cm) }\end{array}$ & $\begin{array}{c}\mathbf{S F} \\
\left(\mathbf{m}^{2}\right)\end{array}$ \\
\hline \multirow[t]{2}{*}{$\mathrm{d} 1$} & Moy & 134,19 & 7,53 & $2,99 \mathrm{~b}$ & 402,78 & 114,92 & 122,52 & $6,15 \mathrm{ab}$ & 17,33 \\
\hline & $\mathrm{CV}$ & 0,07 & 0,05 & 0,08 & 0,06 & 0,03 & 0,09 & 0,09 & 0,14 \\
\hline \multirow[t]{2}{*}{ d2 } & Moy & 136,94 & 7,45 & $3,12 \mathrm{ab}$ & 412,81 & 112,61 & 123,71 & $6,09 \mathrm{ab}$ & 16,97 \\
\hline & $\mathrm{CV}$ & 0,08 & 0,06 & 0,05 & 0,05 & 0,04 & 0,10 & 0,08 & 0,13 \\
\hline \multirow[t]{2}{*}{ d3 } & Moy & 132,62 & 7,58 & $3,03 \mathrm{ab}$ & 412,82 & 114,03 & 121,68 & $5,89 \mathrm{ab}$ & 16,33 \\
\hline & $\mathrm{CV}$ & 0,07 & 0,06 & 0,07 & 0,04 & 0,04 & 0,05 & 0,09 & 0,11 \\
\hline \multirow[t]{2}{*}{$\mathrm{d} 4$} & Moy & 132,78 & 7,38 & $3,02 \mathrm{~b}$ & 415,79 & 113,53 & 122,42 & $6,03 \mathrm{ab}$ & 16,75 \\
\hline & $\mathrm{CV}$ & 0,06 & 0,04 & 0,05 & 0,04 & 0,05 & 0,06 & 0,06 & 0,09 \\
\hline \multirow[t]{2}{*}{ d5 } & Moy & 135,75 & 7,34 & $3,07 \mathrm{ab}$ & 415,08 & 116,17 & 119,97 & $6,23 \mathrm{ab}$ & 17,36 \\
\hline & $\mathrm{CV}$ & 0,08 & 0,04 & 0,06 & 0,06 & 0,04 & 0,05 & 0,06 & 0,10 \\
\hline \multirow[t]{2}{*}{ d6 } & Moy & 135,14 & 7,51 & $3,08 \mathrm{ab}$ & 422,00 & 114,35 & 121,74 & $6,01 \mathrm{ab}$ & 16,72 \\
\hline & $\mathrm{CV}$ & 0,06 & 0,05 & 0,05 & 0,07 & 0,04 & 0,07 & 0,08 & 0,11 \\
\hline \multirow[t]{2}{*}{ d7 } & Moy & 131,68 & 7,35 & $3,00 \mathrm{~b}$ & 403,03 & 114,97 & 116,94 & $6,11 \mathrm{ab}$ & 16,41 \\
\hline & $\mathrm{CV}$ & 0,10 & 0,05 & 0,07 & 0,06 & 0,03 & 0,08 & 0,09 & 0,12 \\
\hline \multirow[t]{2}{*}{$\mathrm{d} 8$} & Moy & 137,11 & 7,23 & $3,05 \mathrm{ab}$ & 405,94 & 114,74 & 120,83 & $5,98 \mathrm{ab}$ & 16,89 \\
\hline & $\mathrm{CV}$ & 0,09 & 0,06 & 0,06 & 0,08 & 0,03 & 0,08 & 0,08 & 0,13 \\
\hline \multirow[t]{2}{*}{ d9 } & Moy & 132,09 & 7,25 & $2,99 \mathrm{~b}$ & 399,72 & 114,69 & 118,54 & $5,88 \mathrm{ab}$ & 15,99 \\
\hline & $\mathrm{CV}$ & 0,09 & 0,06 & 0,05 & 0,06 & 0,04 & 0,08 & 0,06 & 0,11 \\
\hline \multirow[t]{2}{*}{$\mathrm{d} 10$} & Moy & 130,01 & 7,34 & $2,97 \mathrm{~b}$ & 400,94 & 114,53 & 119,97 & $5,84 \mathrm{ab}$ & 16,11 \\
\hline & $\mathrm{CV}$ & 0,11 & 0,07 & 0,06 & 0,06 & 0,04 & 0,08 & 0,10 & 0,16 \\
\hline \multirow[t]{2}{*}{ d11 } & Moy & 128,88 & 7,50 & $3,03 \mathrm{ab}$ & 399,83 & 114,94 & 119,19 & $5,93 \mathrm{ab}$ & 16,32 \\
\hline & $\mathrm{CV}$ & 0,08 & 0,07 & 0,06 & 0,07 & 0,04 & 0,09 & 0,07 & 0,16 \\
\hline d12 & Moy & 133,04 & 7,47 & $3,01 \mathrm{~b}$ & 410,67 & 113,00 & 123,55 & $5,73 \mathrm{~b}$ & 16,05 \\
\hline
\end{tabular}




\begin{tabular}{|c|c|c|c|c|c|c|c|c|c|}
\hline & $\mathrm{CV}$ & 0,06 & 0,06 & 0,08 & 0,06 & 0,04 & 0,07 & 0,07 & 0,13 \\
\hline \multirow[t]{2}{*}{ d13 } & Moy & 132,00 & 7,37 & $3,11 \mathrm{ab}$ & 412,06 & 113,59 & 123,60 & $5,99 \mathrm{ab}$ & 16,83 \\
\hline & $\mathrm{CV}$ & 0,10 & 0,06 & 0,07 & 0,05 & 0,04 & 0,07 & 0,07 & 0,11 \\
\hline \multirow[t]{2}{*}{ d14 } & Moy & 135,06 & 7,52 & $3,04 \mathrm{ab}$ & 418,06 & 112,00 & 127,58 & $5,96 \mathrm{ab}$ & 17,02 \\
\hline & $\mathrm{CV}$ & 0,08 & 0,05 & 0,07 & 0,06 & 0,05 & 0,09 & 0,06 & 0,12 \\
\hline \multirow[t]{2}{*}{ d15 } & Moy & 134,99 & 7,80 & $3,25 \mathrm{a}$ & 419,56 & 116,08 & 123,81 & $6,42 \mathrm{a}$ & 18,47 \\
\hline & $\mathrm{CV}$ & 0,07 & 0,06 & 0,06 & 0,05 & 0,04 & 0,06 & 0,07 & 0,11 \\
\hline \multirow[t]{2}{*}{ d16 } & Moy & 131,71 & 7,38 & $3,11 \mathrm{ab}$ & 411,09 & 113,11 & 123,44 & $5,93 \mathrm{ab}$ & 16,65 \\
\hline & $\mathrm{CV}$ & 0,10 & 0,07 & 0,09 & 0,08 & 0,05 & 0,10 & 0,08 & 0,17 \\
\hline \multirow[t]{2}{*}{ d17 } & Moy & 131,86 & 7,43 & $3,04 \mathrm{ab}$ & 404,62 & 111,94 & 121,88 & $5,88 \mathrm{ab}$ & 16,11 \\
\hline & $\mathrm{CV}$ & 0,08 & 0,06 & 0,07 & 0,06 & 0,04 & 0,08 & 0,09 & 0,16 \\
\hline \multirow[t]{2}{*}{ d18 } & Moy & 126,76 & 7,29 & $3,04 \mathrm{ab}$ & 402,24 & 113,00 & 121,51 & $5,88 \mathrm{ab}$ & 16,20 \\
\hline & $\mathrm{CV}$ & 0,11 & 0,09 & 0,07 & 0,07 & 0,05 & 0,09 & 0,10 & 0,16 \\
\hline
\end{tabular}

Tableau 4: Valeurs moyennes, coefficients de variations des variables agromorphologiques liées aux inflorescences et tests de comparaison chez les hybrides de cocotier étudiés.

\begin{tabular}{|c|c|c|c|c|c|c|c|c|c|}
\hline Descendances & & $\begin{array}{l}\text { ILP } \\
\text { (cm) }\end{array}$ & $\begin{array}{l}\text { ILA } \\
(\mathbf{c m})\end{array}$ & NBE & NBESF & NBEF & $\begin{array}{l}\text { ILE } \\
(\mathbf{c m})\end{array}$ & $\begin{array}{c}\text { ILPRF } \\
(\mathbf{c m})\end{array}$ & NBFF \\
\hline \multirow[t]{2}{*}{$\mathrm{d} 1$} & Moy & 55,43 & 37,28 & $37,45 \mathrm{ab}$ & $18,83 \mathrm{ab}$ & 18,61 & 42,29 & 7,93 & 20,50 \\
\hline & $\mathrm{CV}$ & 0,08 & 0,18 & 0,16 & 0,38 & 0,48 & 0,13 & 0,20 & 0,71 \\
\hline \multirow[t]{2}{*}{$\mathrm{d} 2$} & Moy & 56,63 & 38,00 & $38,56 \mathrm{ab}$ & $15,94 \mathrm{abc}$ & 22,61 & 44,19 & 9,04 & 24,58 \\
\hline & $\mathrm{CV}$ & 0,10 & 0,13 & 0,14 & 0,45 & 0,33 & 0,13 & 0,18 & 0,38 \\
\hline \multirow[t]{2}{*}{$\mathrm{d} 3$} & Moy & 55,25 & 36,30 & $37,24 \mathrm{ab}$ & $19,24 \mathrm{ab}$ & 18,00 & 44,20 & 8,68 & 18,24 \\
\hline & $\mathrm{CV}$ & 0,09 & 0,12 & 0,13 & 0,22 & 0,28 & 0,12 & 0,18 & 0,28 \\
\hline \multirow[t]{2}{*}{$\mathrm{d} 4$} & Moy & 56,59 & 38,42 & $38,15 \mathrm{ab}$ & $15,59 \mathrm{bc}$ & 22,56 & 43,06 & 8,71 & 24,24 \\
\hline & $\mathrm{CV}$ & 0,08 & 0,10 & 0,12 & 0,35 & 0,24 & 0,10 & 0,16 & 0,31 \\
\hline \multirow[t]{2}{*}{ d5 } & Moy & 59,26 & 38,44 & $37,95 \mathrm{ab}$ & $11,53 \mathrm{c}$ & 26,42 & 41,07 & 8,81 & 31,53 \\
\hline & $\mathrm{CV}$ & 0,07 & 0,15 & 0,16 & 0,41 & 0,27 & 0,08 & 0,14 & 0,69 \\
\hline \multirow[t]{2}{*}{ d6 } & Moy & 55,03 & 37,77 & $38,00 \mathrm{ab}$ & $15,44 \mathrm{bc}$ & 22,56 & 42,44 & 8,15 & 24,68 \\
\hline & $\mathrm{CV}$ & 0,09 & 0,13 & 0,08 & 0,38 & 0,31 & 0,12 & 0,14 & 0,45 \\
\hline \multirow[t]{2}{*}{$\mathrm{d} 7$} & Moy & 56,28 & 37,98 & $34,87 \mathrm{ab}$ & $14,36 \mathrm{bc}$ & 20,50 & 42,51 & 8,53 & 21,36 \\
\hline & $\mathrm{CV}$ & 0,11 & 0,14 & 0,14 & 0,38 & 0,34 & 0,11 & 0,15 & 0,39 \\
\hline \multirow[t]{2}{*}{$\mathrm{d} 8$} & Moy & 58,83 & 38,94 & $39,29 \mathrm{ab}$ & $16,96 \mathrm{abc}$ & 23,26 & 43,40 & 8,76 & 23,44 \\
\hline & $\mathrm{CV}$ & 0,08 & 0,12 & 0,14 & 0,36 & 0,24 & 0,12 & 0,14 & 0,26 \\
\hline \multirow[t]{2}{*}{ d9 } & Moy & 57,03 & 37,81 & $37,89 \mathrm{ab}$ & $14,81 \mathrm{abc}$ & 23,09 & 43,45 & 9,33 & 26,67 \\
\hline & $\mathrm{CV}$ & 0,07 & 0,14 & 0,12 & 0,42 & 0,34 & 0,13 & 0,13 & 0,48 \\
\hline \multirow[t]{2}{*}{$\mathrm{d} 10$} & Moy & 53,85 & 36,91 & $37,91 \mathrm{ab}$ & $16,00 \mathrm{abc}$ & 21,91 & 40,90 & 8,15 & 22,91 \\
\hline & $\mathrm{CV}$ & 0,09 & 0,18 & 0,22 & 0,43 & 0,33 & 0,12 & 0,19 & 0,39 \\
\hline \multirow[t]{2}{*}{ d11 } & Moy & 55,02 & 35,62 & $37,23 \mathrm{ab}$ & $16,34 \mathrm{abc}$ & 20,89 & 40,46 & 7,85 & 21,25 \\
\hline & $\mathrm{CV}$ & 0,07 & 0,09 & 0,19 & 0,34 & 0,30 & 0,11 & 0,18 & 0,30 \\
\hline $\mathrm{d} 12$ & Moy & 53,81 & 36,03 & $35,87 \mathrm{ab}$ & $16,25 \mathrm{bc}$ & 19,62 & 41,20 & 7,84 & 20,20 \\
\hline
\end{tabular}




\begin{tabular}{|c|c|c|c|c|c|c|c|c|c|}
\hline & $\mathrm{CV}$ & 0,08 & 0,15 & 0,18 & 0,25 & 0,33 & 0,13 & 0,24 & 0,36 \\
\hline \multirow[t]{2}{*}{ d13 } & Moy & 55,06 & 36,30 & $38,36 \mathrm{ab}$ & $19,71 \mathrm{ab}$ & 18,65 & 43,21 & 8,20 & 19,06 \\
\hline & $\mathrm{CV}$ & 0,08 & 0,16 & 0,11 & 0,21 & 0,31 & 0,11 & 0,14 & 0,33 \\
\hline \multirow[t]{2}{*}{ d14 } & Moy & 57,67 & 39,00 & $36,64 a b$ & $16,92 \mathrm{abc}$ & 19,73 & 46,02 & 9,00 & 21,14 \\
\hline & $\mathrm{CV}$ & 0,06 & 0,13 & 0,12 & 0,23 & 0,23 & 0,10 & 0,11 & 0,30 \\
\hline \multirow[t]{2}{*}{ d15 } & Moy & 55,00 & 37,09 & $40,12 \mathrm{a}$ & 22,62 a & 17,50 & 45,63 & 8,53 & 17,53 \\
\hline & $\mathrm{CV}$ & 0,07 & 0,10 & 0,14 & 0,27 & 0,30 & 0,11 & 0,12 & 0,30 \\
\hline \multirow[t]{2}{*}{ d16 } & Moy & 53,62 & 35,46 & $36,17 \mathrm{ab}$ & $17,56 \mathrm{bc}$ & 18,62 & 41,72 & 7,92 & 19,12 \\
\hline & $\mathrm{CV}$ & 0,11 & 0,17 & 0,14 & 0,40 & 0,50 & 0,15 & 0,21 & 0,57 \\
\hline \multirow[t]{2}{*}{ d17 } & Moy & 55,21 & 40,35 & $39,72 \mathrm{ab}$ & $18,29 \mathrm{abc}$ & 21,44 & 42,79 & 8,28 & 24,57 \\
\hline & $\mathrm{CV}$ & 0,07 & 0,20 & 0,15 & 0,35 & 0,44 & 0,12 & 0,19 & 0,65 \\
\hline \multirow[t]{2}{*}{$\mathrm{d} 18$} & Moy & 55,53 & 37,00 & $34,34 \mathrm{~b}$ & $14,09 \mathrm{bc}$ & 20,25 & 43,55 & 8,67 & 23,92 \\
\hline & $\mathrm{CV}$ & 0,11 & 0,14 & 0,16 & 0,36 & 0,39 & 0,11 & 0,15 & 0,75 \\
\hline
\end{tabular}

Tableau 5: Valeurs moyennes, coefficients de variations des variables agromorphologiques liées à la masse et aux dimensions des noix et tests de comparaison chez les hybrides de cocotier étudiés.

\begin{tabular}{llcccc}
\hline Descendances & & Dp(cm) & Déq $(\mathbf{c m})$ & $\mathbf{d P / d E}$ & MN(g) \\
\hline $\mathrm{d} 1$ & Moy & $19,28 \mathrm{ab}$ & 15,79 & 1,23 & 832,87 \\
& CV & 0,12 & 0,12 & 0,11 & 0,16 \\
$\mathrm{~d} 2$ & Moy & $18,68 \mathrm{ab}$ & 15,45 & 1,23 & 875,00 \\
& CV & 0,08 & 0,13 & 0,12 & 0,23 \\
$\mathrm{~d} 3$ & Moy & $18,42 \mathrm{ab}$ & 14,80 & 1,25 & 799,68 \\
& CV & 0,07 & 0,09 & 0,09 & 0,12 \\
$\mathrm{~d} 4$ & Moy & $17,31 \mathrm{ab}$ & 14,02 & 1,21 & 761,86 \\
& CV & 0,17 & 0,19 & 0,16 & 0,22 \\
$\mathrm{~d} 5$ & Moy & $17,73 \mathrm{~b}$ & 15,42 & 1,17 & 791,67 \\
& CV & 0,08 & 0,13 & 0,11 & 0,19 \\
d6 & Moy & $18,41 \mathrm{ab}$ & 14,87 & 1,25 & 891,35 \\
& CV & 0,08 & 0,11 & 0,10 & 0,15 \\
d7 & Moy & $18,56 \mathrm{ab}$ & 15,28 & 1,23 & 751,39 \\
& CV & 0,08 & 0,09 & 0,11 & 0,20 \\
d8 & Moy & $19,68 \mathrm{ab}$ & 15,45 & 1,28 & 849,62 \\
& CV & 0,08 & 0,10 & 0,08 & 0,15 \\
d9 & Moy & $18,31 \mathrm{ab}$ & 14,92 & 1,24 & 909,47 \\
& CV & 0,15 & 0,14 & 0,11 & 0,31 \\
d10 & Moy & $19,24 \mathrm{ab}$ & 14,67 & 1,33 & 752,24 \\
& CV & 0,08 & 0,12 & 0,09 & 0,21 \\
d11 & Moy & $18,69 \mathrm{ab}$ & 14,33 & 1,32 & 831,25 \\
& CV & 0,10 & 0,10 & 0,11 & 0,20 \\
d12 & Moy & $19,10 \mathrm{ab}$ & 14,42 & 1,34 & 725,35 \\
& CV & 0,10 & 0,08 & 0,08 & 0,20 \\
& & & & &
\end{tabular}




\begin{tabular}{|c|c|c|c|c|c|}
\hline \multirow[t]{2}{*}{$\mathrm{d} 13$} & Moy & $18,94 \mathrm{ab}$ & 15,35 & 1,25 & 799,52 \\
\hline & $\mathrm{CV}$ & 0,09 & 0,10 & 0,09 & 0,22 \\
\hline \multirow[t]{2}{*}{$\mathrm{d} 14$} & Moy & $18,65 \mathrm{ab}$ & 15,49 & 1,22 & 805,83 \\
\hline & $\mathrm{CV}$ & 0,10 & 0,14 & 0,11 & 0,27 \\
\hline \multirow[t]{2}{*}{$\mathrm{d} 15$} & Moy & 19,98 a & 16,13 & 1,29 & 873,66 \\
\hline & $\mathrm{CV}$ & 0,10 & 0,15 & 0,13 & 0,20 \\
\hline \multirow[t]{2}{*}{$\mathrm{d} 16$} & Moy & $18,99 \mathrm{ab}$ & 15,16 & 1,27 & 863,72 \\
\hline & $\mathrm{CV}$ & 0,06 & 0,10 & 0,09 & 0,20 \\
\hline \multirow[t]{2}{*}{$\mathrm{d} 17$} & Moy & $18,65 \mathrm{ab}$ & 14,75 & 1,28 & 793,89 \\
\hline & $\mathrm{CV}$ & 0,08 & 0,10 & 0,09 & 0,19 \\
\hline \multirow[t]{2}{*}{$\mathrm{d} 18$} & Moy & $17,39 \mathrm{~b}$ & 14,17 & 1,24 & 766,99 \\
\hline & $\mathrm{CV}$ & 0,12 & 0,14 & 0,10 & 0,29 \\
\hline
\end{tabular}

Tableau 6 : Valeurs moyennes, coefficients de variations des variables agromorphologiques liées à la masse des composantes des noix et tests de comparaison chez les hybrides de cocotier étudiés.

\begin{tabular}{llcccccc}
\hline Descendances & & MB $(\mathbf{g})$ & MCQ $(\mathbf{g})$ & MALB $(\mathbf{g})$ & ME $(\mathbf{g})$ & MCP(g) & Q $(\%)$ \\
\hline d1 & Moy & $236,11 \mathrm{ab}$ & 124,63 & 301,30 & 170,83 & 159,20 & 23,97 \\
& CV & 0,16 & 0,15 & 0,14 & 0,31 & 0,19 & 0,11 \\
d2 & Moy & $230,58 \mathrm{ab}$ & 134,20 & 315,80 & 194,42 & 162,33 & 23,90 \\
& CV & 0,25 & 0,19 & 0,22 & 0,38 & 0,23 & 0,14 \\
d3 & Moy & $205,77 \mathrm{~b}$ & 116,35 & 315,71 & 161,86 & 170,76 & 26,79 \\
& CV & 0,17 & 0,14 & 0,12 & 0,22 & 0,19 & 0,16 \\
d4 & Moy & $206,73 \mathrm{~b}$ & 118,91 & 297,28 & 138,94 & 154,18 & 24,79 \\
& CV & 0,23 & 0,18 & 0,15 & 0,59 & 0,21 & 0,15 \\
d5 & Moy & $192,50 \mathrm{~b}$ & 121,43 & 307,10 & 170,64 & 157,40 & 25,23 \\
& CV & 0,15 & 0,19 & 0,19 & 0,38 & 0,23 & 0,14 \\
d6 & Moy & $240,58 \mathrm{ab}$ & 136,25 & 320,80 & 193,72 & 163,39 & 23,54 \\
& CV & 0,28 & 0,19 & 0,15 & 0,34 & 0,16 & 0,15 \\
d7 & Moy & $205,76 \mathrm{~b}$ & 113,89 & 295,14 & 136,60 & 154,32 & 25,17 \\
& CV & 0,21 & 0,19 & 0,20 & 0,42 & 0,22 & 0,16 \\
d8 & Moy & $246,32 \mathrm{ab}$ & 128,79 & 316,67 & 157,92 & 166,31 & 24,00 \\
& CV & 0,20 & 0,16 & 0,20 & 0,35 & 0,21 & 0,16 \\
d9 & Moy & $250,83 \mathrm{ab}$ & 132,20 & 325,38 & 223,86 & 165,21 & 25,21 \\
& CV & 0,43 & 0,23 & 0,23 & 0,35 & 0,25 & 0,25 \\
d10 & Moy & $193,14 \mathrm{~b}$ & 117,15 & 290,22 & 154,81 & 160,72 & 27,29 \\
& CV & 0,35 & 0,26 & 0,18 & 0,40 & 0,15 & 0,13 \\
d11 & Moy & $217,42 \mathrm{ab}$ & 122,73 & 318,56 & 172,54 & 163,16 & 24,90 \\
& CV & 0,23 & 0,18 & 0,17 & 0,35 & 0,21 & 0,18 \\
d12 & Moy & $210,76 \mathrm{~b}$ & 107,99 & 278,30 & 128,30 & 148,52 & 25,06 \\
\hline & & & & & &
\end{tabular}




\begin{tabular}{llcccccc}
\hline \multirow{4}{*}{ d13 } & CV & 0,25 & 0,17 & 0,19 & 0,44 & 0,19 & 0,16 \\
& Moy & $231,89 \mathrm{ab}$ & 118,04 & 302,15 & 150,00 & 164,74 & 25,26 \\
$\mathrm{~d} 14$ & CV & 0,26 & 0,22 & 0,20 & 0,53 & 0,19 & 0,11 \\
& Moy & $217,56 \mathrm{ab}$ & 128,22 & 296,22 & 163,83 & 156,18 & 24,69 \\
$\mathrm{~d} 15$ & CV & 0,24 & 0,23 & 0,22 & 0,52 & 0,20 & 0,18 \\
& Moy & $291,82 \mathrm{a}$ & 125,89 & 309,23 & 146,73 & 167,89 & 23,00 \\
$\mathrm{~d} 16$ & CV & 0,25 & 0,28 & 0,22 & 0,43 & 0,24 & 0,14 \\
& Moy & $239,41 \mathrm{ab}$ & 131,84 & 301,15 & 191,32 & 145,51 & 21,30 \\
$\mathrm{~d} 17$ & CV & 0,22 & 0,22 & 0,22 & 0,34 & 0,31 & 0,20 \\
& Moy & $220,14 \mathrm{ab}$ & 119,78 & 299,11 & 154,86 & 160,20 & 25,17 \\
$\mathrm{~d} 18$ & CV & 0,24 & 0,18 & 0,12 & 0,37 & 0,17 & 0,11 \\
& Moy & $202,56 \mathrm{~b}$ & 123,24 & 277,08 & 164,10 & 142,32 & 23,75 \\
& CV & 0,35 & 0,34 & 0,24 & 0,52 & 0,29 & 0,20 \\
\hline
\end{tabular}

Les moyennes indexées par la même lettre ne sont pas significativement différentes au seuil de 5\% (test de Newman Keuls).

Tableau 7: Analyse en composantes principales : Valeurs propres et pourcentage de variation des variables quantitatives sur les deux axes principaux d'une analyse en composantes principales.

\begin{tabular}{lcc}
\hline Composantes & Axe 1 & Axe 2 \\
\hline Valeurs propres & 4,09 & 1,16 \\
\hline Variances expliquées & 58,37 & 16,62 \\
\hline Variances Cumulées (\%) & 58,37 & 74,99 \\
\hline Variables définissant les axes & C20 $(-0,84)$ & EP $(0,67)$ \\
et leurs corrélations & EP $(-0,86)$ & DP $(-0,51)$ \\
& LGF $(-0,70)$ & \\
& NBE $(-0,69)$ & \\
& NBESF $(-0,76)$ & \\
& DP $(-0,69)$ & \\
& MB $(-0,79)$ & \\
\hline
\end{tabular}

Tableau 8: Distance de Mahalanobis et test de Fischer entre les classes constituées par la Classification Ascendante Hiérarchique

\begin{tabular}{cccc}
\hline & \multicolumn{1}{c}{ Classe 1 } & Classe 2 & Classe 3 \\
\hline Classe 1 & $\mathrm{~F}=0,43 ; \mathrm{P}=0,87$ & $\mathrm{D}=0,99$ & $\mathrm{D}=6,50$ \\
Classe 2 & $\mathrm{~F}=5,19 ; \mathrm{P}<0,01$ & $\mathrm{~F}=2,98 ; \mathrm{P}=0,02$ & \\
Classe 3 & & \\
\hline $\mathrm{D}=$ Distance de Mahalanobis au carré & & \\
$\mathrm{F}=$ La statistique du test de Fischer & & \\
$\mathrm{P}=$ La probabilité du test de Fischer & &
\end{tabular}




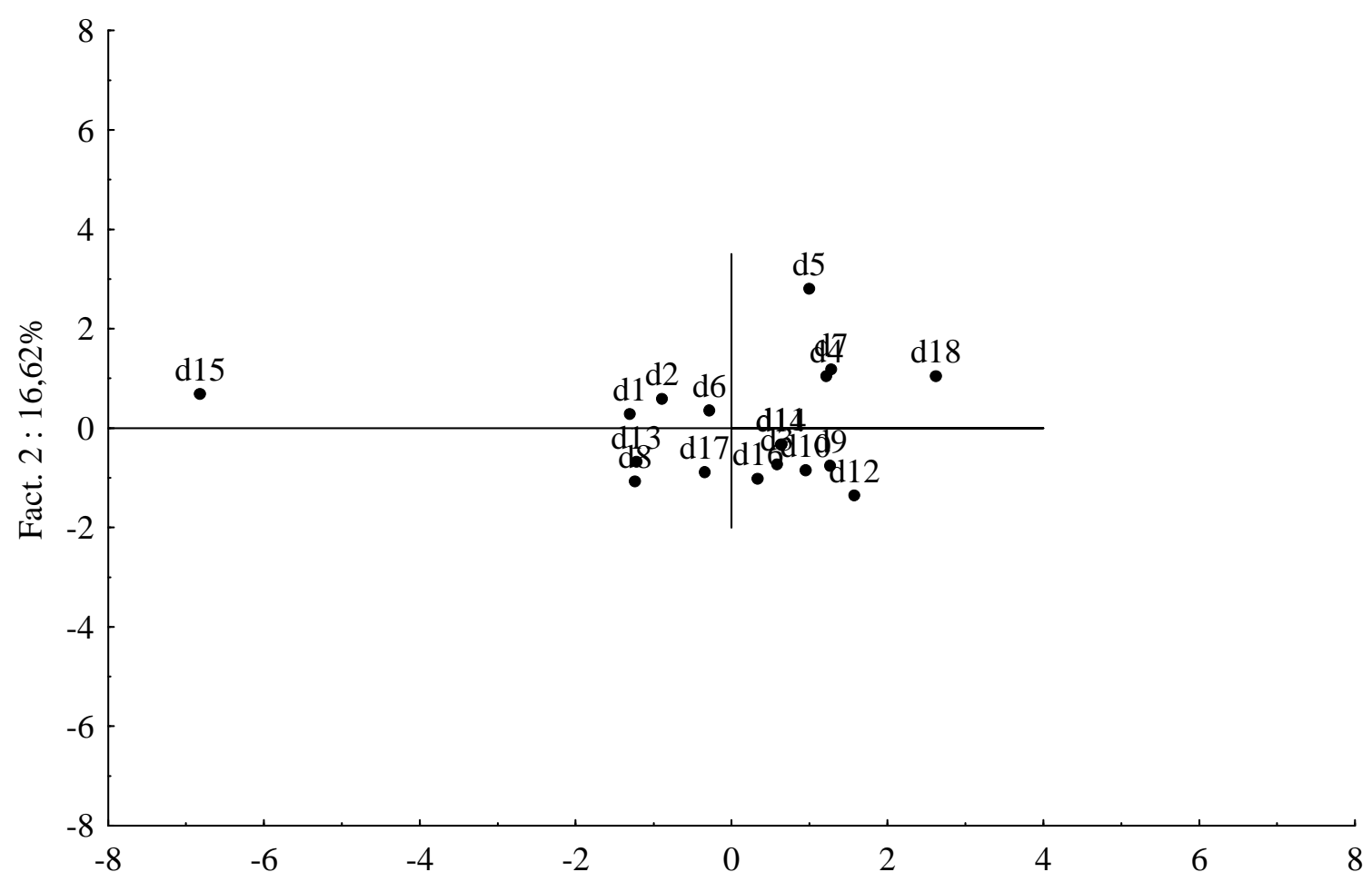

Fact. $1: 58,37 \%$

Figure 2: Distribution des descendances dans le plan 1-2 selon une analyse en composantes principales.

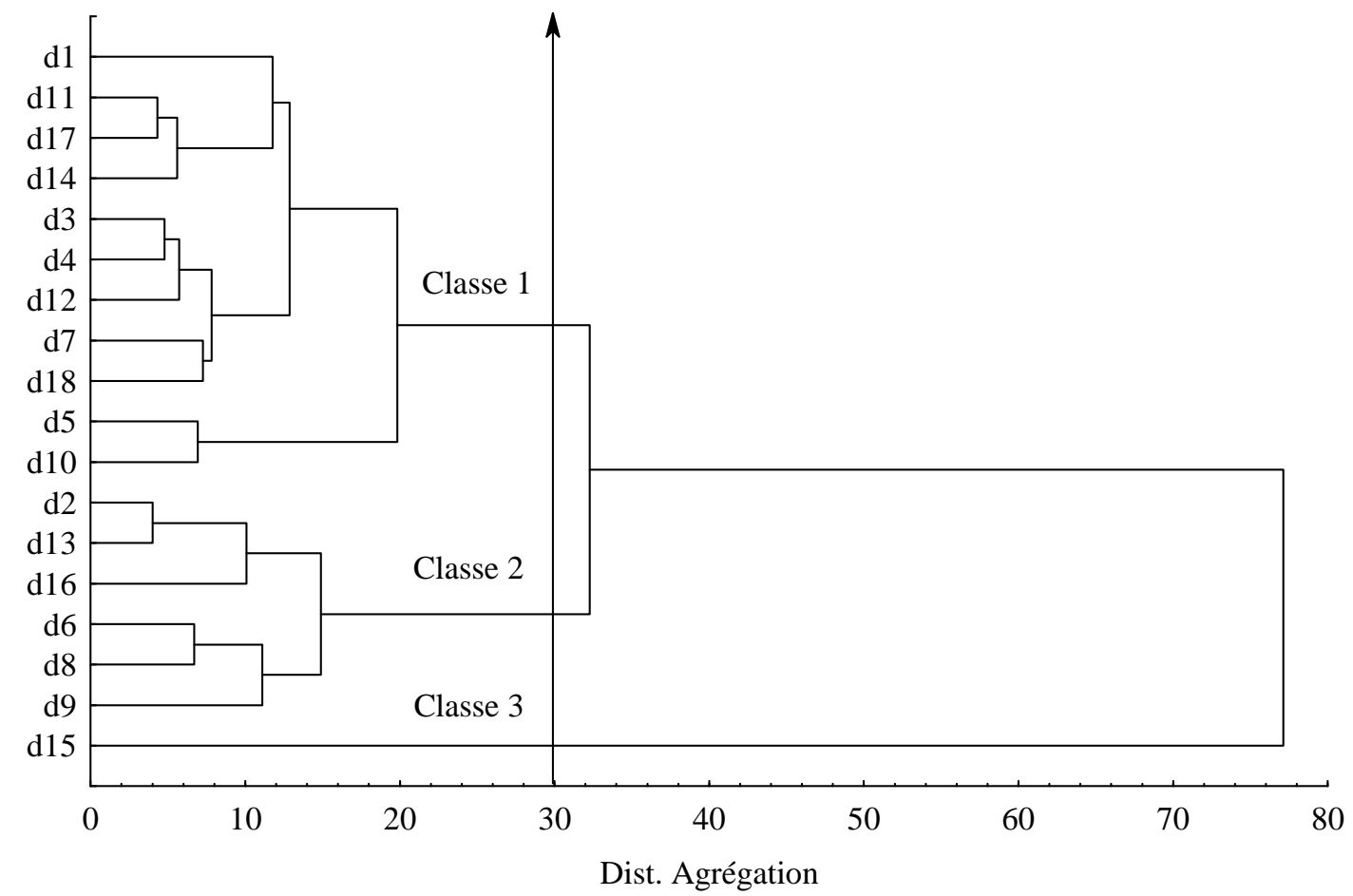

Figure 3: Dendrogramme UPGMA des descendances hybrides étudiées. 


\section{DISCUSSION}

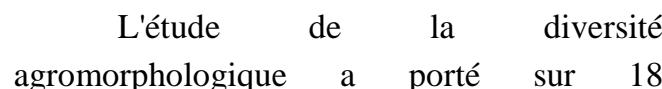
descendances hybrides NJM x GVT. Les résultats des statistiques élémentaires portant sur les descripteurs agromorphologiques à la station de recherche Marc Delorme en Côte d'Ivoire, ont montré dans l'ensemble de forte ressemblance entre les descendances. Le Nain Jaune Malaisie, du fait de son système de reproduction autogame préférentiel, est très homogène (Perera et al., 2000; Konan, 2008). L'effet maternel étant dominant chez le cocotier (Koffi, 2008; Konan et al., 2010), le géniteur femelle aurait contribué à l'homogénéisation des descendances hybrides NJM x GVT. La variabilité intra-population est plus élevée chez les cocotiers grands que chez les nains. Des études antérieures ont montré l'hétérogénéité des cocotiers grands et les hybrides grand $\mathrm{x}$ grand ainsi que l'homogénéité des cocotiers nain et les hybrides nain $\mathrm{x}$ nain (De Nucé et Wuidart, 1981; Singh and Sharma, 2005; Been, 2005). Quelques caractères ont permis cependant d'observer une diversité inter et intradescendance. De telles diversités résulteraient de la variabilité qui existe au niveau des géniteurs Grand de Vanuatu (Calvez et al., 1985; Konan et al., 2007).

Le GVT étant hétérozygote et le NJM homozygote (Bourdeix et al., 2005), la diversité intra-descendance résulterait d'une ségrégation d'allèles du géniteur GVT. La variabilité inter et intra-descendance pourrait être la cause de la variation dans la tolérance au jaunissement comme l'a indiqué Konan (2008). Naturellement, la couleur des fruits permet de distinguer trois types de Grand de Vanuatu dont les rouges, verts et les bruns (Batugal et al., 2010). L'hypothèse d'une relation entre la couleur des fruits et la tolérance au jaunissement mortel n'est pas à exclure. Cependant l'étude des caractères quantitatifs est nécessaire pour l'analyse des
QTLs (Quantitative Trait Loci) intervenant dans la tolérance aux maladies. Il serait donc judicieux d'associer aux caractères quantitatifs mesurés ici des descripteurs qualitatifs à intérêt agronomique qui permettront certainement de mieux affiner la description des descendances.

$$
\begin{array}{ccr}
\text { L'analyse de la diversité } \\
\text { agromorphologique des }
\end{array}
$$
hybrides NJM x GVT nous a conduits ainsi à la structuration en deux groupes distincts. Il s'agit d'une part des arbres robustes composés des descendances $\mathrm{d} 15$ et d'autre part des autres descendances qui sont relativement moins robustes. Le géniteur GVT15 abriterait des gènes spécifiques qu'il aurait transmis à ces descendances (d15). Les descendances d15 ont les plus gros stipes. Ces descendances auraient donc une puissance racinaire leur permettant de résister plus aux grands vents (De Nuce et Wuidart, 1982). Aussi elles seraient relativement plus tolérantes à la sécheresse que les autres (Konan et al., 2006). La sécheresse tout comme les feux de brousse peuvent affaiblir les arbres et augmenter leur sensibilité aux attaques de parasites. Les arbres aux bulbes volumineux possèdent un système racinaire très développé rendant également plus efficace l'absorption hydrique (Konan, 1997). Les arbres aux bulbes racinaires plus volumineux seraient relativement plus tolérants à la sécheresse. Le système racinaire peu donc intervenir comme un des mécanismes de défense du cocotier pour maintenir l'alimentation en eau régulière pendant la saison sèche (Cintra et al., 1993). Les périodes prolongés de sècheresse ainsi que les déficits en eau sévères font apparaître des troubles végétatifs pouvant provoquer la mort de l'arbre (Rajagopal et al., 1990; Rajagopal et al., 2005). Pour éviter toute nuance entre les symptômes du jaunissement mortel et ceux du déficit hydrique, la sélection de descendance relativement tolérante à la sècheresse pour des évaluations 
épidémiologique du jaunissement mortel pourrait contribuer à une bonne appréciation des individus tolérants à la maladie.

Les largeurs élevées des folioles des d15 pourraient correspondre à une forte activité photosynthétique et par conséquent une bonne production. Le nombre d'épillets statistiquement élevé chez ces descendances milite également pour une forte production de noix. Ce caractère, en effet, a été identifié par Konan et al. (2006) comme un indice de production. Cependant, un nombre trop élevé d'épillet pourrait influencer négativement le nombre de noix amenées à maturité. La compétition inter-noix pour l'espace disponible pourrait provoquer la chute précoce de plusieurs noix.

\section{Conclusion}

Cette étude a été conduite pour évaluer la diversité agromorphologique des descendances hybrides NJM x GVT. A cet effet, 30 descripteurs ont été mesurés sur 18 descendances codées de d1 à d18. Les observations ont porté sur le stipe, les feuilles, les inflorescences et les fruits.

Il ressort de nos travaux qu'il existe une diversité inter et intra-descendance. Les différences fondamentales observées entre les descendances sont liées à la circonférence du stipe à $20 \mathrm{~cm}$ (C20), à l'épaisseur du pétiole (EP), à la largeur de foliole (LGF), au nombre d'épillet sur l'inflorescence (NBE), au nombre d'épillet sans fleur femelle (NBESF), au diamètre polaire (DP) et à la masse de la bourre (MB). Les descendances d15 se sont montrées plus robustes et ont eu des valeurs de C20 (134,52 cm), EP (3,25 cm), LGF (6,42 cm), NBE (40), NBESF (23), DP (20,03 cm) et MB $(291,82 \mathrm{~g})$ supérieures aux autres. Les variables hauteur du stipe (HS), nombre d'épillet avec fleur femelle (NBEF), le nombre de fleur femelle (NBFF), longueur sur 11 cicatrices foliaires (L11CF) et la masse de l'eau (ME) se sont montrées très hétérogènes au niveau intra-descendance. Elles ont des coefficients de variation qui fluctuent entre 0,20 et 0,75 . Le géniteur G15 qui donne des descendances robustes présentant des aptitudes favorables à une bonne production est à sélectionner pour produire des hybrides NJM x GVT tolérants et haut producteurs.

\section{REFERENCES}

Assa RRA, Konan JL, Nemlin J, Prades A, Agbo N, Sie RS. 2006. Diagnostic de la cocoteraie paysanne du littoral ivoirien. Sci. Nat., 3(2): 113-120.

Baudouin L, Philippe R, Quaicoe R, Dery S, Dollet M. 2009. General overview of genetic research and experimentation on coconut varieties tolerant/resistant to Lethal Yellowing. OCL., 16(2): 127-131.

Batugal P, Bourdeix R, Olivier J, George M. 2010. Catalogue of Conservation Coconut Germplasm. COGENT, Bioversity International, Regional Office for Asia, the Pacific and Oceania, Serdang: Selangor Darul Ehsan, Malaysia; 399.

Baudouin L, Santos G. 2005. Morphometric methodes of determining diversity in coconut. In Coconut Genetic Resources, Batugal P, Ramanatha Rao V, Olivier J (eds). International Plant Genetic Resources Institute: Selangor Darul Ehsan, Malaisie; 209 - 224.

Been B. 2005. Coconut hybrids trials in Jamaica. In Coconut Hybrids for Smallholders, Batugal P, Dante B, Olivier J (eds). Common Fund for Commodities / International Plant Genetic Resources Institute (CFC / IPGRI): Amsterdam, Netherlands; 98-110.

Bourdeix R, Konan JL, N'cho YP. 2005. Cocotier, Guide des Variétés Traditionnelles et Améliorées. Coproduction CIRAD/CNRA, édition diversiflora: Montpellier, France; 58. 
Calvez C, Julia JF, DE Nuce de LM. 1985. L'amélioration du cocotier au Vanuatu et son intérêt pour la région pacifique. Oléagineux, 40(10): 477-490.

Cinta FLD, Passos EE, Da Silva Leal M. 1993. Evaluation de la distribution du système racinaire de cultivars de cocotier grand. Oléagineux, 48(11): 453-461.

Dery SK, Owusu Nipah J, Andoh-Mensah E, Nuertey BN, Nkansah Poku J, Arthur R, Philippe R. 2005. On-farm evaluation of the coconut hybrid, Malayan yellow dwarf $x$ Vanuatu tall for tolerance to the lethal yellowing disease of coconut in Ghana. Cord., 21(1): 50-56.

De Nuce de LM, Wuidart W. 1982. L'observation des caractéristiques de développement végétatif, de floraison et de production chez le cocotier. Oléagineux, 37(6): 291-300.

De Nuce de LM, Wuidart W. 1981. Les cocotiers Grands à port-bouet. 2. Grand Rennell, Grand Salomon, Grand Thaillande, Grand Nouvelles-Hébrides. Oléagineux, 36(7): 353-366.

Koffi EBZ. 2008. Etude des caractéristiques agromorphologiques des hybrides améliorés de cocotier (Cocos nucifera L.) grand en Côte d'Ivoire. Diplôme d'Etudes Approfondies, Université d'AboboAdjamé, Côte d'Ivoire, 47.

Konan JL. 2005. Status of coconut genetic resources research in Côte d'Ivoire. In Coconut Genetic Resources, Batugal p, Ramanatha Rao V, Olivier J (eds). International Plant Genetic Resources Institute: Selangor Darul Ehsan, Malaisie; 654-660.

Konan KJN. 2008. Evaluation de la diversité agromorphologique et moléculaire de la collection internationale de cocotier (Cocos nucifera L.) en Côte d'Ivoire. Thèse de doctorat unique. Université de Cocody, Côte d'Ivoire, p. 150.

Konan JL, Sié SR, N'guetta SP, Lekadou TT,
Allou K. 2010. Assessment of vegetative growth and production of new improved coconut (Cocos nucifera L.) hybrids. Journal of Applied Biosciences, 26: 16641674.

Konan JL, Bourdeix R, Sangare A, Mondeil F. 2006. Caractérisation de quelques cultivars de cocotier (Cocos nucifera L.) tolérant à la sécheresse en Côte d'Ivoire. Agronomie Africaine, 18(2): 145-156.

Konan KJN, Koffi KE, Konan JL, Lebrun P, Dery SK, Sangare A. 2007. Microsatellite gene diversity in coconut (Cocos nucifera L.) accessions resistants to lethal yellowing disease. African Journal of Biotechnology, 6(4): 341-347.

Konan KLL. 1997. Etude de la tolerance à la secheresse chez le cocotier (Cocos nucifera L.): Evaluation de quelques caractères biologiques et physiologiques. Thèse de doctorat $3^{\text {ème }}$ cycle. Université de Cocody, Côte d'Ivoire, p. 110.

Oropeza C, Escamilla JA, Mora G, Zizumbo D, Harrison NA. 2005. Coconut lethal yellowing. In Coconut Genetic Resources, Batugal p, Ramanatha Rao V, Olivier J (eds). International Plant Genetic Resources Institute: Selangor Darul Ehsan, Malaisie; 349-363.

Perera L, Russell JR, Provan J, Powell W. 2000. Use of microsatellite DNA markers to investigate the level of genetic diversity and population genetic structure of coconut (Cocos nucifera L.). Genome, 43: $15-21$.

Quaicoe RN, Dery SK, Philippe R, Baudouin L, Nipah JO, Nkansah-Poku J, Arthur R, Dare D, Yankey E N, Pilet F, Dollet M. 2009. Resistance screening trials on coconut varieties to Cape Saint Paul Wilt Disease in Ghana. OCL, 16(2): 132-136.

Rajagopal V, Kasturibai KV, Kumar N. 2005. Breeding for drought tolerance in coconut: status and potentials. In Coconut Genetic Resources, Batugal p, Ramanatha 
Rao V, Olivier J (eds). International Plant Genetic Resources Institute: Selangor Darul Ehsan, Malaisie; 282 - 301.

Rajagopal V, Kasturibai KV, Voleti SR. 1990. Sélection des génotypes de cocotier pour la tolérance à la sécheresse. Oléagineux, 45(5): 215-223.

Sangare A, DE Taffin G, DE Franqueville H, Arkhust ED, Pomier M. 1992. Le jaunissement mortel du cocotier au Ghana. Premier résultats sur le comportement au champ du matériel végétal. Oléagineux, 47(12): 699-704.

Singh G, Sharma M. 2005. Results of coconut hybrid trials by United Plantations Sdn Bhd, Malaysia. In Coconut Hybrids for Smallholders, Batugal P, Dante B, Olivier
J, (eds). Common Fund for Commodities / International Plant Genetic Resources Institute (CFC / IPGRI): Amsterdam, Netherlands; 132-150.

Tie Bi Y. 1984. Contribution à l'étude des sols sableux de la basse Côte d'Ivoire, cultivée en cocotiers et définition des seuils d'utilisation de la fumure phosphatée. Thèse de Doctorat $3^{\text {ème }}$ cycle. Université de Cocody, Côte d'Ivoire, p. 182.

Van der vossen HAM, Chipungahelo GSE. 2007. Cocos nucifera L. In Vegetable oils/Oléagineux. Van der Vossen HAM, Mkamilo GS (eds). PROTA : Wageningen, Pays Bas; 62-72. 